\title{
Research Article \\ Convergence Theorems for Common Fixed Points of Nonself Asymptotically Quasi-Non-Expansive Mappings
}

\author{
Chao Wang and Jinghao Zhu \\ Department of Applied Mathematics, Tongji University, Shanghai 200092, China \\ Correspondence should be addressed to Chao Wang, wangchaoxj20002000@yahoo.com.cn
}

Received 1 April 2008; Revised 12 June 2008; Accepted 19 July 2008

Recommended by Simeon Reich

We introduce a new three-step iterative scheme with errors. Several convergence theorems of this scheme are established for common fixed points of nonself asymptotically quasi-non-expansive mappings in real uniformly convex Banach spaces. Our theorems improve and generalize recent known results in the literature.

Copyright (C) 2008 C. Wang and J. Zhu. This is an open access article distributed under the Creative Commons Attribution License, which permits unrestricted use, distribution, and reproduction in any medium, provided the original work is properly cited.

\section{Introduction}

Let $K$ be a nonempty closed convex subset of real normed linear space $E$. Recall that a mapping $T: K \rightarrow K$ is called asymptotically nonexpansive if there exists a sequence $\left\{r_{n}\right\} \subset$ $[0, \infty)$, with $\lim _{n \rightarrow \infty} r_{n}=0$ such that $\left\|T^{n} x-T^{n} y\right\| \leq\left(1+r_{n}\right)\|x-y\|$, for all $x, y \in K$ and $n \geq 1$. Moreover, it is uniformly L-Lipschitzian if there exists a constant $L>0$ such that $\left\|T^{n} x-T^{n} y\right\| \leq L\|x-y\|$, for all $x, y \in K$ and each $n \geq 1$. Denote and define by $F(T)=\{x \in K: T x=x\}$ the set of fixed points of $T$. Suppose $F(T) \neq \varnothing$. A mapping $T$ is called asymptotically quasi-non-expansive if there exists a sequence $\left\{r_{n}\right\} \subset[0, \infty)$, with $\lim _{n \rightarrow \infty} r_{n}=0$ such that $\left\|T^{n} x-p\right\| \leq\left(1+r_{n}\right)\|x-p\|$, for all $x, y \in K, p \in F(T)$, and $n \geq 1$.

It is clear from the above definitions that an asymptotically nonexpansive mapping must be uniformly L-Lipschitzian as well as asymptotically quasi-non-expansive, but the converse does not hold. Iterative technique for asymptotically nonexpansive self-mapping in Hilbert spaces and Banach spaces including Mann-type and Ishikawa-type iteration processes has been studied extensively by many authors; see, for example, [1-6].

Recently, Chidume et al. [7] have introduced the concept of nonself asymptotically nonexpansive mappings, which is the generalization of asymptotically nonexpansive mappings. Similarly, the concept of nonself asymptotically quasi-non-expansive mappings 
can also be defined as the generalization of asymptotically quasi-non-expansive mappings and nonself asymptotically nonexpansive mappings. These mappings are defined as follows.

Definition 1.1. Let $K$ be a nonempty closed convex subset of real normed linear space $E$, let $P: E \rightarrow K$ be the nonexpansive retraction of $E$ onto $K$, and let $T: K \rightarrow E$ be a nonself mapping.

(i) $T$ is said to be a nonself asymptotically nonexpansive mapping if there exists a sequence $\left\{r_{n}\right\} \subset[0, \infty)$, with $\lim _{n \rightarrow \infty} r_{n}=0$ such that

$$
\left\|T(P T)^{n-1} x-T(P T)^{n-1} y\right\| \leq\left(1+r_{n}\right)\|x-y\|,
$$

for all $x, y \in K$ and $n \geq 1$.

(ii) $T$ is said to be a nonself uniformly $L$-Lipschitzian mapping if there exists a constant $L>0$ such that

$$
\left\|T(P T)^{n-1} x-T(P T)^{n-1} y\right\| \leq L\|x-y\|,
$$

for all $x, y \in K$ and $n \geq 1$.

(iii) $T$ is said to be a nonself asymptotically quasi-non-expansive mapping if $F(T) \neq \varnothing$ and there exists a sequence $\left\{r_{n}\right\} \subset[0, \infty)$, with $\lim _{n \rightarrow \infty} r_{n}=0$ such that

$$
\left\|T(P T)^{n-1} x-p\right\| \leq\left(1+r_{n}\right)\|x-p\|,
$$

for all $x, y \in K, p \in F(T)$, and $n \geq 1$.

By studying the following iteration process (Mann-type iteration):

$$
x_{1} \in K, \quad x_{n+1}=P\left(\left(1-\alpha_{n}\right) x_{n}+\alpha_{n} T(P T)^{n-1} x_{n}\right), \quad \forall n \geq 1,
$$

where $\left\{\alpha_{n}\right\} \subset[0,1]$, Chidume et al. [7] obtained many convergence theorems for the fixed points of nonself asymptotically nonexpansive mapping $T$. Later on, Wang [8] generalized the iteration process (1.4) as follows (Ishikawa-type iteration):

$$
\begin{gathered}
x_{1} \in K, \\
x_{n+1}=P\left(\left(1-\alpha_{n}\right) x_{n}+\alpha_{n} T_{1}\left(P T_{1}\right)^{n-1} y_{n}\right), \\
y_{n}=P\left(\left(1-\beta_{n}\right) x_{n}+\beta_{n} T_{2}\left(P T_{2}\right)^{n-1} x_{n}\right), \quad \forall n \geq 1
\end{gathered}
$$

where $T_{1}, T_{2}: K \rightarrow E$ are nonself asymptotically nonexpansive mappings and $\left\{\alpha_{n}\right\},\left\{\beta_{n}\right\} \subset$ $[0,1]$. Also, he got several convergence theorems of the iterative scheme (1.5) under proper conditions.

In 2000, Noor [9] first introduced a three-step iterative sequence and studied the approximate solutions of variational inclusion in Hilbert spaces by using the techniques of updating the solution and the auxiliary principle. Glowinski and Tallec [10] showed that the three-step iterative schemes perform better than the Mann-type and Ishikawa-type iterative schemes. On the other hand, $\mathrm{Xu}$ and Noor [11] introduced and studied a three-step scheme to approximate fixed points of asymptotically nonexpansive mappings in Banach spaces. Cho et al. [12] and Plubtieng et al. [13] extended the work of $\mathrm{Xu}$ and Noor to the threestep iterative scheme with errors, and gave weak and strong convergence theorems for asymptotically nonexpansive mappings in Banach spaces. 
Inspired and motivated by these facts, a new class of three-step iterative schemes with errors, for three nonself asymptotically quasi-non-expansive mappings, is introduced and studied in this paper. This scheme can be viewed as an extension for (1.4), (1.5), and others. This scheme is defined as follows.

Let $K$ be a nonempty convex subset of real normed linear space $X$, let $P: E \rightarrow K$ be the nonexpansive retraction of $E$ onto $K$, and let $T_{1}, T_{2}, T_{3}: K \rightarrow E$ be three nonself asymptotically quasi-non-expansive mappings. Compute the sequences $\left\{x_{n}\right\},\left\{y_{n}\right\}$, and $\left\{z_{n}\right\}$ by

$$
\begin{gathered}
x_{1} \in K, \\
x_{n+1}=P\left(\alpha_{n} T_{1}\left(P T_{1}\right)^{n-1} y_{n}+\beta_{n} x_{n}+\gamma_{n} w_{n}\right), \\
y_{n}=P\left(\alpha_{n}^{\prime} T_{2}\left(P T_{2}\right)^{n-1} z_{n}+\beta_{n}^{\prime} x_{n}+\gamma_{n}^{\prime} v_{n}\right), \\
z_{n}=P\left(\alpha_{n}^{\prime \prime} T_{3}\left(P T_{3}\right)^{n-1} x_{n}+\beta_{n}^{\prime \prime} x_{n}+\gamma_{n}^{\prime \prime} u_{n}\right), \quad \forall n \geq 1
\end{gathered}
$$

where $\left\{\alpha_{n}\right\},\left\{\alpha_{n}^{\prime}\right\},\left\{\alpha_{n}^{\prime \prime}\right\},\left\{\beta_{n}\right\},\left\{\beta_{n}^{\prime}\right\},\left\{\beta_{n}^{\prime \prime}\right\},\left\{\gamma_{n}\right\},\left\{\gamma_{n}^{\prime}\right\}$, and $\left\{\gamma_{n}^{\prime \prime}\right\}$ are real sequences in $[0,1]$ with $\alpha_{n}+\beta_{n}+\gamma_{n}=\alpha_{n}^{\prime}+\beta_{n}^{\prime}+\gamma_{n}^{\prime}=\alpha_{n}^{\prime \prime}+\beta_{n}^{\prime \prime}+\gamma_{n}^{\prime \prime}=1$, and $\left\{u_{n}\right\},\left\{v_{n}\right\}$, and $\left\{w_{n}\right\}$ are bounded sequences in $K$.

Remark 1.2. (i) If $T_{1}=T_{2}=T_{3}:=T, \gamma_{n}=\gamma_{n}^{\prime}=\gamma_{n}^{\prime \prime}=0$, and $\alpha_{n}^{\prime}=\alpha_{n}^{\prime \prime}=0$, then scheme (1.6) reduces to the Mann-type iteration (1.4).

(ii) If $T_{2}=T_{3}, \gamma_{n}=\gamma_{n}^{\prime}=\gamma_{n}^{\prime \prime}=0$, and $\alpha_{n}^{\prime \prime}=0$, then scheme (1.6) reduces to the Ishikawatype iteration (1.5).

(iii) If $T_{1}, T_{2}$, and $T_{3}$ are three self-asymptotically nonexpansive mappings, then scheme (1.6) reduces to the three-step iteration with errors defined by [12,13], and others.

The purpose of this paper is to study the iterative sequences (1.6) to converge to a common fixed point of three nonself asymptotically quasi-non-expansive mappings in real uniformly convex Banach spaces. Our results extend and improve the corresponding results in $[5,7,8,11-13]$, and many others.

\section{Preliminaries and lemmas}

In this section, we first recall some well-known definitions.

A real Banach space $E$ is said to be uniformly convex if the modulus of convexity of $E$ :

$$
\delta_{E}(\varepsilon)=\inf \left\{1-\frac{\|x+y\|}{2}:\|x\|=\|y\|=1,\|x-y\|=\varepsilon\right\}>0,
$$

for all $0<\varepsilon \leq 2$ (i.e., $\delta_{E}(\varepsilon)$ is a function $\left.(0,2] \rightarrow(0,1)\right)$.

A subset $K$ of $E$ is said to be a retract if there exists continuous mapping $P: E \rightarrow K$ such that $P x=x$, for all $x \in K$, and every closed convex subset of a uniformly convex Banach space is a retract. A mapping $P: E \rightarrow E$ is said to be a retraction if $P^{2}=P$.

A mapping $T: K \rightarrow E$ with $F(T) \neq \varnothing$ is said to satisfy condition (A) (see [14]) if there exists a nondecreasing function $f:[0, \infty) \rightarrow[0, \infty)$ with $f(0)=0$, for all $r \in(0, \infty)$, such that

$$
\|x-T x\| \geq f(d(x, F(T))),
$$

for all $x \in K$, where $d(x, F(T))=\inf \left\{\left\|x-x^{*}\right\|: x^{*} \in F(T)\right\}$.

We modify this condition for three mappings $T_{1}, T_{2}, T_{3}: K \rightarrow E$ as follows. Three mappings $T_{1}, T_{2}, T_{3}: K \rightarrow E$, where $K$ is a subset of $E$, are said to satisfy condition (B) if there 
exist a real number $\alpha>0$ and a nondecreasing function $f:[0, \infty) \rightarrow[0, \infty)$ with $f(0)=0$, for all $r \in(0, \infty)$, such that

$$
\left\|x-T_{1} x\right\| \geq \alpha f(d(x, F)) \quad \text { or } \quad\left\|x-T_{2} x\right\| \geq \alpha f(d(x, F)) \quad \text { or } \quad\left\|x-T_{3} x\right\| \geq \alpha f(d(x, F)),
$$

for all $x \in K$, where $F=F\left(T_{1}\right) \cap F\left(T_{2}\right) \cap F\left(T_{3}\right) \neq \varnothing$. Note that condition (B) reduces to condition (A) when $T_{1}=T_{2}=T_{3}$ and $\alpha=1$.

A mapping $T: K \rightarrow E$ is said to be semicompact if, for any sequence $\left\{x_{n}\right\}$ in $K$ such that $\left\|x_{n}-T x_{n}\right\| \rightarrow 0(n \rightarrow \infty)$, there exists subsequence $\left\{x_{n_{j}}\right\}$ of $\left\{x_{n}\right\}$ such that $\left\{x_{n_{j}}\right\}$ converges strongly to $x^{*} \in K$.

Next we state the following useful lemmas.

Lemma 2.1 (see [5]). Let $\left\{a_{n}\right\},\left\{b_{n}\right\}$, and $\left\{c_{n}\right\}$ be sequences of nonnegative real numbers satisfying the inequality

$$
a_{n+1} \leq\left(1+c_{n}\right) a_{n}+b_{n}, \quad \forall n \geq 1 .
$$

If $\sum_{n=1}^{\infty} c_{n}<\infty$ and $\sum_{n=1}^{\infty} b_{n}<\infty$, then $\lim _{n \rightarrow \infty} a_{n}$ exists.

Lemma 2.2 (see [15]). Let $E$ be a real uniformly convex Banach space and $0 \leq k \leq t_{n} \leq q<1$, for all positive integer $n \geq 1$. Suppose that $\left\{x_{n}\right\}$ and $\left\{y_{n}\right\}$ are two sequences of $E$ such that $\limsup _{n \rightarrow \infty}\left\|x_{n}\right\| \leq r, \limsup _{n \rightarrow \infty}\left\|y_{n}\right\| \leq r$, and $\lim _{n \rightarrow \infty}\left\|t_{n} x_{n}+\left(1-t_{n}\right) y_{n}\right\|=r$ hold, for some $r \geq 0$; then $\lim _{n \rightarrow \infty}\left\|x_{n}-y_{n}\right\|=0$.

\section{Main results}

In this section, we will prove the strong convergence of the iteration scheme (1.6) to a common fixed point of nonself asymptotically quasi-non-expansive mappings $T_{1}, T_{2}$, and $T_{3}$. We first prove the following lemmas.

Lemma 3.1. Let $K$ be a nonempty closed convex subset of a real normed linear space $E$. Let $T_{1}, T_{2}, T_{3}$ : $K \rightarrow E$ be nonself asymptotically quasi-non-expansive mappings with sequences $\left\{r_{n}^{(i)}\right\}$ such that $\sum_{n=1}^{\infty} r_{n}^{(i)}<\infty$, for all $i=1,2,3$. Suppose that $\left\{x_{n}\right\}$ is defined by (1.6) with $\sum_{n=1}^{\infty} \gamma_{n}<\infty, \sum_{n=1}^{\infty} \gamma_{n}^{\prime}<$ $\infty$, and $\sum_{n=1}^{\infty} \gamma_{n}^{\prime \prime}<\infty$. If $F=F\left(T_{1}\right) \cap F\left(T_{2}\right) \cap F\left(T_{3}\right) \neq \varnothing$, then $\lim _{n \rightarrow \infty}\left\|x_{n}-p\right\|$ exists, for all $p \in F$.

Proof. Let $p \in F$. Since $\left\{u_{n}\right\},\left\{v_{n}\right\}$, and $\left\{w_{n}\right\}$ are bounded sequences in $K$, therefore there exists $M>0$ such that

$$
M=\max \left\{\sup _{n \geq 1}\left\|u_{n}-p\right\|, \sup _{n \geq 1}\left\|v_{n}-p\right\|, \sup _{n \geq 1}\left\|w_{n}-p\right\|\right\} .
$$

Let $r_{n}=\max \left\{r_{n}^{(1)}, r_{n}^{(2)}, r_{n}^{(3)}\right\}$ and $k_{n}=\max \left\{\gamma_{n}, \gamma_{n}^{\prime}, \gamma_{n}^{\prime \prime}\right\}$. Then $\sum_{n=1}^{\infty} r_{n}<\infty$ and $\sum_{n=1}^{\infty} k_{n}<\infty$. By (1.6), we have

$$
\begin{aligned}
\left\|x_{n+1}-p\right\| & =\left\|P\left[\alpha_{n} T_{1}\left(P T_{1}^{n-1}\right) y_{n}+\beta_{n} x_{n}+\gamma_{n} w_{n}\right]-P(p)\right\| \\
& \leq\left\|\alpha_{n} T_{1}\left(P T_{1}^{n-1}\right) y_{n}+\beta_{n} x_{n}+\gamma_{n} w_{n}-\left(\alpha_{n}+\beta_{n}+\gamma_{n}\right) p\right\| \\
& \leq\left\|\alpha_{n}\left[T_{1}\left(P T_{1}^{n-1}\right) y_{n}-p\right]+\beta_{n}\left(x_{n}-p\right)+\gamma_{n}\left(w_{n}-p\right)\right\| \\
& \leq \alpha_{n}\left(1+r_{n}\right)\left\|y_{n}-p\right\|+\beta_{n}\left\|x_{n}-p\right\|+k_{n}\left\|w_{n}-p\right\|, \\
\left\|y_{n}-p\right\| & =\left\|P\left[\alpha_{n}^{\prime} T_{2}\left(P T_{2}^{n-1}\right) z_{n}+\beta_{n}^{\prime} x_{n}+\gamma_{n}^{\prime} v_{n}\right]-P(p)\right\| \\
& \leq\left\|\alpha_{n}^{\prime} T_{2}\left(P T_{2}^{n-1}\right) z_{n}+\beta_{n}^{\prime} x_{n}+\gamma_{n}^{\prime} v_{n}-\left(\alpha_{n}^{\prime}+\beta_{n}^{\prime}+\gamma_{n}^{\prime}\right) p\right\| \\
& \leq \alpha_{n}^{\prime}\left(1+r_{n}\right)\left\|z_{n}-p\right\|+\beta_{n}^{\prime}\left\|x_{n}-p\right\|+k_{n}\left\|v_{n}-p\right\|,
\end{aligned}
$$


and similarly, we also have

$$
\left\|z_{n}-p\right\| \leq \alpha_{n}^{\prime \prime}\left(1+r_{n}\right)\left\|x_{n}-p\right\|+\beta_{n}^{\prime \prime}\left\|x_{n}-p\right\|+k_{n}\left\|u_{n}-p\right\|
$$

Substituting (3.4) into (3.3), we obtain

$$
\begin{aligned}
\left\|y_{n}-p\right\| \leq & \alpha_{n}^{\prime}\left(1+r_{n}\right)\left[\alpha_{n}^{\prime \prime}\left(1+r_{n}\right)\left\|x_{n}-p\right\|+\beta_{n}^{\prime \prime}\left\|x_{n}-p\right\|+k_{n}\left\|u_{n}-p\right\|\right] \\
& +\beta_{n}^{\prime}\left\|x_{n}-p\right\|+k_{n}\left\|v_{n}-p\right\| \\
\leq & \alpha_{n}^{\prime} \alpha_{n}^{\prime \prime}\left(1+r_{n}\right)^{2}\left\|x_{n}-p\right\|+\alpha_{n}^{\prime} \beta_{n}^{\prime \prime}\left(1+r_{n}\right)\left\|x_{n}-p\right\|+\beta_{n}^{\prime}\left\|x_{n}-p\right\| \\
& +\alpha_{n}^{\prime} k_{n}\left(1+r_{n}\right)\left\|u_{n}-p\right\|+k_{n}\left\|v_{n}-p\right\| \\
\leq & \left(1-\beta_{n}^{\prime}-\gamma_{n}^{\prime}\right) \alpha_{n}^{\prime \prime}\left(1+r_{n}\right)^{2}\left\|x_{n}-p\right\|+\left(1-\beta_{n}^{\prime}-\gamma_{n}^{\prime}\right) \beta_{n}^{\prime \prime}\left(1+r_{n}\right)\left\|x_{n}-p\right\| \\
& +\beta_{n}^{\prime}\left\|x_{n}-p\right\|+k_{n}\left(1+r_{n}\right)\left\|u_{n}-p\right\|+k_{n}\left\|v_{n}-p\right\| \\
\leq & \left(1-\beta_{n}^{\prime}-\gamma_{n}^{\prime}\right)\left(\alpha_{n}^{\prime \prime}+\beta_{n}^{\prime \prime}\right)\left(1+r_{n}\right)^{2}\left\|x_{n}-p\right\|+\beta_{n}^{\prime}\left\|x_{n}-p\right\|+m_{n} \\
\leq & \left(1-\beta_{n}^{\prime}\right)\left(1+r_{n}\right)^{2}\left\|x_{n}-p\right\|+\beta_{n}^{\prime}\left(1+r_{n}\right)^{2}\left\|x_{n}-p\right\|+m_{n} \\
\leq & \left(1+r_{n}\right)^{2}\left\|x_{n}-p\right\|+m_{n},
\end{aligned}
$$

where $m_{n}=k_{n}\left(2+r_{n}\right) M$. Since $\sum_{n=1}^{\infty} r_{n}<\infty$ and $\sum_{n=1}^{\infty} k_{n}<\infty$, then $\sum_{n=1}^{\infty} m_{n}<\infty$. Substituting (3.5) into (3.2), we have

$$
\begin{aligned}
\left\|x_{n+1}-p\right\| & \leq \alpha_{n}\left(1+r_{n}\right)\left[\left(1+r_{n}^{2}\right)\left\|x_{n}-p\right\|+m_{n}\right]+\beta_{n}\left\|x_{n}-p\right\|+\gamma_{n}\left\|w_{n}-p\right\| \\
& \leq\left[\alpha_{n}\left(1+r_{n}\right)^{3}+\beta_{n}\right]\left\|x_{n}-p\right\|+\alpha_{n}\left(1+r_{n}\right) m_{n}+\gamma_{n}\left\|w_{n}-p\right\| \\
& \leq\left(\alpha_{n}+\beta_{n}\right)\left(1+r_{n}\right)^{3}\left\|x_{n}-p\right\|+\left(1+r_{n}\right) m_{n}+k_{n}\left\|w_{n}-p\right\| \\
& \leq\left(1+r_{n}\right)^{3}\left\|x_{n}-p\right\|+\left(1+r_{n}\right) m_{n}+k_{n} M \\
& \leq\left(1+c_{n}\right)\left\|x_{n}-p\right\|+b_{n},
\end{aligned}
$$

where $c_{n}=\left(1+r_{n}\right)^{3}-1$ and $b_{n}=\left(1+r_{n}\right) m_{n}+k_{n} M$. Since $\sum_{n=1}^{\infty} r_{n}<\infty, \sum_{n=1}^{\infty} k_{n}<\infty$, and $\sum_{n=1}^{\infty} m_{n}<\infty$, then $\sum_{n=1}^{\infty} c_{n}<\infty$ and $\sum_{n=1}^{\infty} b_{n}<\infty$. It follows from Lemma 2.1 that $\lim _{n \rightarrow \infty}\left\|x_{n}-p\right\|$ exists. This completes the proof.

Lemma 3.2. Let $K$ be a nonempty closed convex subset of a real uniformly convex Banach space E. Let $T_{1}, T_{2}, T_{3}: K \rightarrow$ E be uniformly L-Lipschitzian nonself asymptotically quasi-non-expansive mappings with sequences $\left\{r_{n}^{(i)}\right\}$ such that $\sum_{n=1}^{\infty} r_{n}^{(i)}<\infty$, for all $i=1,2,3$. Suppose that $\left\{x_{n}\right\}$ is defined by (1.6) with $\sum_{n=1}^{\infty} \gamma_{n}<\infty, \sum_{n=1}^{\infty} \gamma_{n}^{\prime}<\infty$, and $\sum_{n=1}^{\infty} \gamma_{n}^{\prime \prime}<\infty$, where $\alpha_{n}$, $\alpha_{n}^{\prime}$, and $\alpha_{n}^{\prime \prime}$ are three sequences in $[\varepsilon, 1-\varepsilon]$, for some $\varepsilon>0$. If $F=F\left(T_{1}\right) \cap F\left(T_{2}\right) \cap F\left(T_{3}\right) \neq \varnothing$, then

$$
\lim _{n \rightarrow \infty}\left\|x_{n}-T_{1} x_{n}\right\|=\lim _{n \rightarrow \infty}\left\|x_{n}-T_{2} x_{n}\right\|=\lim _{n \rightarrow \infty}\left\|x_{n}-T_{3} x_{n}\right\|=0 .
$$

Proof. For any $p \in F$, by Lemma 3.1, we see that $\lim _{n \rightarrow \infty}\left\|x_{n}-p\right\|$ exists. Assume $\lim _{n \rightarrow \infty} \| x_{n}-$ $p \|=a$, for some $a \geq 0$. For all $n \geq 1$, let $r_{n}=\max \left\{r_{n}^{(1)}, r_{n}^{(2)}, r_{n}^{(3)}\right\}$ and $k_{n}=\max \left\{\gamma_{n}, \gamma_{n}^{\prime}, \gamma_{n}^{\prime \prime}\right\}$. 
Then, $\sum_{n=1}^{\infty} r_{n}<\infty$ and $\sum_{n=1}^{\infty} k_{n}<\infty$. From (3.5), we have

$$
\left\|y_{n}-p\right\| \leq\left(1+r_{n}\right)^{2}\left\|x_{n}-p\right\|+m_{n}
$$

Taking limsup $\operatorname{sum}_{n \rightarrow \infty}$ on both sides in (3.8), since $\sum_{n=1}^{\infty} r_{n}<\infty$ and $\sum_{n=1}^{\infty} m_{n}<\infty$, we obtain

$$
\limsup _{n \rightarrow \infty}\left\|y_{n}-p\right\| \leq \limsup _{n \rightarrow \infty}\left\|x_{n}-p\right\|=\lim _{n \rightarrow \infty}\left\|x_{n}-p\right\|=a
$$

so that

$$
\limsup _{n \rightarrow \infty}\left\|T_{1}\left(P T_{1}\right)^{n-1} y_{n}-p\right\| \leq \limsup _{n \rightarrow \infty}\left(1+r_{n}\right)\left\|y_{n}-p\right\|=\limsup _{n \rightarrow \infty}\left\|y_{n}-p\right\| \leq a
$$

Next consider

$$
\left\|T_{1}\left(P T_{1}\right)^{n-1} y_{n}-p+\gamma_{n}\left(w_{n}-x_{n}\right)\right\| \leq\left\|T_{1}\left(P T_{1}\right)^{n-1} y_{n}-p\right\|+k_{n}\left\|w_{n}-x_{n}\right\| .
$$

Since $\lim _{n \rightarrow \infty} k_{n}=0$, we have

$$
\limsup _{n \rightarrow \infty}\left\|T_{1}\left(P T_{1}\right)^{n-1} y_{n}-p+\gamma_{n}\left(w_{n}-x_{n}\right)\right\| \leq a
$$

In addition,

$$
\left\|x_{n}-p+\gamma_{n}\left(w_{n}-x_{n}\right)\right\| \leq\left\|x_{n}-p\right\|+k_{n}\left\|w_{n}-x_{n}\right\| .
$$

This implies that

$$
\limsup _{n \rightarrow \infty}\left\|x_{n}-p+\gamma_{n}\left(w_{n}-x_{n}\right)\right\| \leq a
$$

Further, observe that

$$
\begin{aligned}
a & =\lim _{n \rightarrow \infty}\left\|x_{n}-p\right\| \\
= & \lim _{n \rightarrow \infty}\left\|\alpha_{n} T_{1}\left(P T_{1}\right)^{n-1} y_{n}+\beta_{n} x_{n}+\gamma_{n} w_{n}-p\right\| \\
= & \lim _{n \rightarrow \infty}\left\|\alpha_{n} T_{1}\left(P T_{1}\right)^{n-1} y_{n}+\left(1-\alpha_{n}\right) x_{n}-\gamma_{n} x_{n}+\gamma_{n} w_{n}-\left(1-\alpha_{n}\right) p-\alpha_{n} p\right\| \\
= & \lim _{n \rightarrow \infty} \| \alpha_{n} T_{1}\left(P T_{1}\right)^{n-1} y_{n}-\alpha_{n} p+\alpha_{n} \gamma_{n} w_{n}-\alpha_{n} \gamma_{n} x_{n}+\left(1-\alpha_{n}\right) x_{n} \\
& \quad-\left(1-\alpha_{n}\right) p-\gamma_{n} x_{n}+\gamma_{n} w_{n}-\alpha_{n} \gamma_{n} w_{n}+\alpha_{n} \gamma_{n} x_{n} \| \\
= & \lim _{n \rightarrow \infty}\left\|\alpha_{n}\left[T_{1}\left(P T_{1}\right)^{n-1} y_{n}-p+\gamma_{n}\left(w_{n}-x_{n}\right)\right]+\left(1-\alpha_{n}\right)\left[x_{n}-p+\gamma_{n}\left(w_{n}-x_{n}\right)\right]\right\| .
\end{aligned}
$$

By Lemma 2.2, (3.12), (3.14), and (3.15), we have

$$
\lim _{n \rightarrow \infty}\left\|T_{1}\left(P T_{1}\right)^{n-1} y_{n}-x_{n}\right\|=0
$$


Next we will prove that $\lim _{n \rightarrow \infty}\left\|T_{2}\left(P T_{2}\right)^{n-1} z_{n}-x_{n}\right\|=0$. Since

$$
\begin{aligned}
\left\|x_{n}-p\right\| & \leq\left\|T_{1}\left(P T_{1}\right)^{n-1} y_{n}-x_{n}\right\|+\left\|T_{1}\left(P T_{1}\right)^{n-1} y_{n}-p\right\| \\
& \leq\left\|T_{1}\left(P T_{1}\right)^{n-1} y_{n}-x_{n}\right\|+\left(1+r_{n}\right)\left\|y_{n}-p\right\|
\end{aligned}
$$

and $\lim _{n \rightarrow \infty}\left\|T_{1}\left(P T_{1}\right)^{n-1} y_{n}-x_{n}\right\|=0=\lim _{n \rightarrow \infty} r_{n}$, we obtain

$$
a=\lim _{n \rightarrow \infty}\left\|x_{n}-p\right\| \leq \liminf _{n \rightarrow \infty}\left\|y_{n}-p\right\| .
$$

Thus, it follows from (3.10) and (3.18) that

$$
\lim _{n \rightarrow \infty}\left\|y_{n}-p\right\|=a
$$

On the other hand, from (3.4), we have

$$
\begin{aligned}
\left\|z_{n}-p\right\| & \leq\left[\alpha_{n}^{\prime \prime}\left(1+r_{n}\right)+\beta_{n}^{\prime \prime}\right]\left\|x_{n}-p\right\|+k_{n}\left\|u_{n}-p\right\| \\
& \leq\left(1+r_{n}\right)\left\|x_{n}-p\right\|+k_{n}\left\|u_{n}-p\right\| .
\end{aligned}
$$

By boundedness of the sequence $\left\{u_{n}\right\}$ and by $\lim _{n \rightarrow \infty} r_{n}=\lim _{n \rightarrow \infty} k_{n}=0$, we have

$$
\limsup _{n \rightarrow \infty}\left\|z_{n}-p\right\| \leq \underset{n \rightarrow \infty}{\limsup }\left\|x_{n}-p\right\|=a
$$

so that

$$
\underset{n \rightarrow \infty}{\limsup }\left\|T_{2}\left(P T_{2}\right)^{n-1} z_{n}-p\right\| \leq \limsup _{n \rightarrow \infty}\left(1+r_{n}\right)\left\|z_{n}-p\right\| \leq a .
$$

Next consider

$$
\left\|T_{2}\left(P T_{2}\right)^{n-1} z_{n}-p+\gamma_{n}^{\prime}\left(v_{n}-x_{n}\right)\right\| \leq\left\|T_{2}\left(P T_{2}\right)^{n-1} z_{n}-p\right\|+k_{n}\left\|v_{n}-x_{n}\right\| .
$$

Thus, we have

$$
\begin{gathered}
\limsup _{n \rightarrow \infty}\left\|T_{2}\left(P T_{2}\right)^{n-1} z_{n}-p+\gamma_{n}^{\prime}\left(v_{n}-x_{n}\right)\right\| \leq a, \\
\left\|x_{n}-p+\gamma_{n}^{\prime}\left(v_{n}-x_{n}\right)\right\| \leq\left\|x_{n}-p\right\|+k_{n}\left\|v_{n}-x_{n}\right\| .
\end{gathered}
$$

This implies that

$$
\underset{n \rightarrow \infty}{\limsup }\left\|x_{n}-p+\gamma_{n}^{\prime}\left(v_{n}-x_{n}\right)\right\| \leq a .
$$

Note that

$$
\begin{aligned}
a & =\lim _{n \rightarrow \infty}\left\|y_{n}-p\right\| \\
& =\lim _{n \rightarrow \infty}\left\|\alpha_{n}^{\prime} T_{2}\left(P T_{2}\right)^{n-1} z_{n}+\beta_{n}^{\prime} x_{n}+\gamma_{n}^{\prime} v_{n}-p\right\| \\
& =\lim _{n \rightarrow \infty}\left\|\alpha_{n}^{\prime}\left[T_{2}\left(P T_{2}\right)^{n-1} z_{n}-p+\gamma_{n}^{\prime}\left(v_{n}-x_{n}\right)\right]+\left(1-\alpha_{n}^{\prime}\right)\left[x_{n}-p+\gamma_{n}^{\prime}\left(v_{n}-x_{n}\right)\right]\right\| .
\end{aligned}
$$


It follows from Lemma 2.2, (3.24), and (3.25) that

$$
\lim _{n \rightarrow \infty}\left\|T_{2}\left(P T_{2}\right)^{n-1} z_{n}-x_{n}\right\|=0 .
$$

Similarly, by using the same argument as in the proof above, we obtain

$$
\lim _{n \rightarrow \infty}\left\|T_{3}\left(P T_{3}\right)^{n-1} x_{n}-x_{n}\right\|=0 \text {. }
$$

Hence,

$$
\lim _{n \rightarrow \infty}\left\|T_{1}\left(P T_{1}\right)^{n-1} y_{n}-x_{n}\right\|=\lim _{n \rightarrow \infty}\left\|T_{2}\left(P T_{2}\right)^{n-1} z_{n}-x_{n}\right\|=\lim _{n \rightarrow \infty}\left\|T_{3}\left(P T_{3}\right)^{n-1} x_{n}-x_{n}\right\|=0,
$$

and this implies that

$$
\left\|x_{n+1}-x_{n}\right\| \leq \alpha_{n}\left\|T_{1}\left(P T_{1}\right)^{n-1} y_{n}-x_{n}\right\|+k_{n}\left\|w_{n}-x_{n}\right\| \longrightarrow 0 \quad \text { as } n \longrightarrow \infty .
$$

Since $T_{1}$ is uniformly $L$-Lipschitzian mapping, then we have

$$
\begin{aligned}
& \left\|T_{1}\left(P T_{1}\right)^{n-1} x_{n}-x_{n}\right\| \\
& \quad \leq\left\|T_{1}\left(P T_{1}\right)^{n-1} x_{n}-T_{1}\left(P T_{1}\right)^{n-1} y_{n}\right\|+\left\|T_{1}\left(P T_{1}\right)^{n-1} y_{n}-x_{n}\right\| \\
& \quad \leq L\left\|x_{n}-y_{n}\right\|+\left\|T_{1}\left(P T_{1}\right)^{n-1} y_{n}-x_{n}\right\| \\
& \quad \leq L\left\|x_{n}-\alpha_{n}^{\prime} T_{2}\left(P T_{2}\right)^{n-1} z_{n}-\beta_{n}^{\prime} x_{n}-\gamma_{n}^{\prime} v_{n}\right\|+\left\|T_{1}\left(P T_{1}\right)^{n-1} y_{n}-x_{n}\right\| \\
& \quad \leq L \alpha_{n}^{\prime}\left\|T_{2}\left(P T_{2}\right)^{n-1} z_{n}-x_{n}\right\|+L k_{n}\left\|v_{n}-x_{n}\right\|+\left\|T_{1}\left(P T_{1}\right)^{n-1} y_{n}-x_{n}\right\| \longrightarrow 0 \quad \text { as } n \longrightarrow \infty, \\
& \left\|x_{n}-T_{1} x_{n}\right\| \\
& \quad \leq\left\|x_{n+1}-x_{n}\right\|+\left\|x_{n+1}-T_{1}\left(P T_{1}\right)^{n} x_{n+1}\right\|+\left\|T_{1}\left(P T_{1}\right)^{n} x_{n+1}-T_{1}\left(P T_{1}\right)^{n} x_{n}\right\|+\left\|T_{1}\left(P T_{1}\right)^{n} x_{n}-T_{1} x_{n}\right\| \\
& \quad \leq\left\|x_{n+1}-x_{n}\right\|+\left\|x_{n+1}-T_{1}\left(P T_{1}\right)^{n} x_{n+1}\right\|+L\left\|x_{n+1}-x_{n}\right\|+L\left\|T_{1}\left(P T_{1}\right)^{n-1} x_{n}-x_{n}\right\| .
\end{aligned}
$$

It follows from (3.30), (3.31), and (3.32) that

$$
\lim _{n \rightarrow \infty}\left\|x_{n}-T_{1} x_{n}\right\|=0 \text {. }
$$

Next consider

$$
\begin{aligned}
& \left\|T_{2}\left(P T_{2}\right)^{n-1} x_{n}-x_{n}\right\| \\
& \quad \leq\left\|T_{2}\left(P T_{2}\right)^{n-1} x_{n}-T_{2}\left(P T_{2}\right)^{n-1} z_{n}\right\|+\left\|T_{2}\left(P T_{2}\right)^{n-1} z_{n}-x_{n}\right\| \\
& \quad \leq L\left\|x_{n}-z_{n}\right\|+\left\|T_{2}\left(P T_{2}\right)^{n-1} z_{n}-x_{n}\right\| \\
& \quad \leq L \alpha_{n}^{\prime \prime}\left\|T_{3}\left(P T_{3}\right)^{n-1} x_{n}-x_{n}\right\|+L k_{n}\left\|u_{n}-x_{n}\right\|+\left\|T_{2}\left(P T_{2}\right)^{n-1} z_{n}-x_{n}\right\| \longrightarrow 0 \quad \text { as } n \longrightarrow \infty,
\end{aligned}
$$

$$
\begin{aligned}
& \left\|x_{n}-T_{2} x_{n}\right\| \\
& \quad \leq\left\|x_{n+1}-x_{n}\right\|+\left\|x_{n+1}-T_{2}\left(P T_{2}\right)^{n} x_{n+1}\right\|+\left\|T_{2}\left(P T_{2}\right)^{n} x_{n+1}-T_{2}\left(P T_{2}\right)^{n} x_{n}\right\|+\left\|T_{2}\left(P T_{2}\right)^{n} x_{n}-T_{2} x_{n}\right\| \\
& \quad \leq\left\|x_{n+1}-x_{n}\right\|+\left\|x_{n+1}-T_{2}\left(P T_{2}\right)^{n} x_{n+1}\right\|+L\left\|x_{n+1}-x_{n}\right\|+L\left\|T_{2}\left(P T_{2}\right)^{n-1} x_{n}-x_{n}\right\| .
\end{aligned}
$$


It follows from (3.30), (3.34), and (3.35) that

$$
\lim _{n \rightarrow \infty}\left\|x_{n}-T_{2} x_{n}\right\|=0 .
$$

Finally, we consider

$$
\begin{aligned}
& \left\|x_{n}-T_{3} x_{n}\right\| \\
& \quad \leq\left\|x_{n+1}-x_{n}\right\|+\left\|x_{n+1}-T_{3}\left(P T_{3}\right)^{n} x_{n+1}\right\|+\left\|T_{3}\left(P T_{3}\right)^{n} x_{n+1}-T_{3}\left(P T_{3}\right)^{n} x_{n}\right\|+\left\|T_{3}\left(P T_{3}\right)^{n} x_{n}-T_{3} x_{n}\right\| \\
& \quad \leq\left\|x_{n+1}-x_{n}\right\|+\left\|x_{n+1}-T_{3}\left(P T_{3}\right)^{n} x_{n+1}\right\|+L\left\|x_{n+1}-x_{n}\right\|+L\left\|T_{3}\left(P T_{3}\right)^{n-1} x_{n}-x_{n}\right\| .
\end{aligned}
$$

It follows from (3.29), (3.30), and (3.37) that

$$
\lim _{n \rightarrow \infty}\left\|x_{n}-T_{3} x_{n}\right\|=0
$$

Therefore,

$$
\lim _{n \rightarrow \infty}\left\|x_{n}-T_{1} x_{n}\right\|=\lim _{n \rightarrow \infty}\left\|x_{n}-T_{2} x_{n}\right\|=\lim _{n \rightarrow \infty}\left\|x_{n}-T_{3} x_{n}\right\|=0 .
$$

This completes the proof.

Now, we give our main theorems of this paper.

Theorem 3.3. Let $K$ be a nonempty closed convex subset of a real uniformly convex Banach space $E$. Let $T_{1}, T_{2}, T_{3}: K \rightarrow$ E be uniformly L-Lipschitzian and nonself asymptotically quasi-non-expansive mappings with sequences $\left\{r_{n}^{(i)}\right\}$ such that $\sum_{n=1}^{\infty} r_{n}^{(i)}<\infty$, for all $i=1,2,3$, satisfying condition (B). Suppose that $\left\{x_{n}\right\}$ is defined by (1.6) with $\sum_{n=1}^{\infty} \gamma_{n}<\infty, \sum_{n=1}^{\infty} \gamma_{n}^{\prime}<\infty$, and $\sum_{n=1}^{\infty} \gamma_{n}^{\prime \prime}<\infty$, where $\alpha_{n}, \alpha_{n}^{\prime}$, and $\alpha_{n}^{\prime \prime}$ are three sequences in $[\varepsilon, 1-\varepsilon]$, for some $\varepsilon>0$. If $F=F\left(T_{1}\right) \cap F\left(T_{2}\right) \cap F\left(T_{3}\right) \neq \varnothing$, then $\left\{x_{n}\right\}$ converges strongly to a common fixed point of $T_{1}, T_{2}$, and $T_{3}$.

Proof. It follows from Lemma 3.2 that $\lim _{n \rightarrow \infty}\left\|x_{n}-T_{1} x_{n}\right\|=\lim _{n \rightarrow \infty}\left\|x_{n}-T_{2} x_{n}\right\|=\lim _{n \rightarrow \infty} \| x_{n}-$ $T_{3} x_{n} \|=0$. Since $T_{1}, T_{2}$, and $T_{3}$ satisfy condition (B), we have $\lim _{n \rightarrow \infty} d\left(x_{n}, F\right)=0$.

From Lemma 3.1 and the proof of Qihou [5], we can obtain that $\left\{x_{n}\right\}$ is a Cauchy sequence in $K$. Assume that $\lim _{n \rightarrow \infty} x_{n}=p \in K$. Since $\lim _{n \rightarrow \infty}\left\|x_{n}-T_{1} x_{n}\right\|=\lim _{n \rightarrow \infty} \| x_{n}-$ $T_{2} x_{n}\left\|=\lim _{n \rightarrow \infty}\right\| x_{n}-T_{3} x_{n} \|=0$, by the continuity of $T_{1}, T_{2}$, and $T_{3}$, we have $p \in F$, that is, $p$ is a common fixed point of $T_{1}, T_{2}$, and $T_{3}$. This completes the proof.

Corollary 3.4. Let $K$ be a nonempty closed convex subset of a real uniformly convex Banach space E. Let $T_{1}, T_{2}, T_{3}: K \rightarrow E$ be nonself asymptotically nonexpansive mappings with sequences $\left\{r_{n}^{(i)}\right\}$ such that $\sum_{n=1}^{\infty} r_{n}^{(i)}<\infty$, for all $i=1,2,3$, satisfying condition (B). Suppose that $\left\{x_{n}\right\}$ is defined by (1.6) with $\sum_{n=1}^{\infty} \gamma_{n}<\infty, \sum_{n=1}^{\infty} \gamma_{n}^{\prime}<\infty$, and $\sum_{n=1}^{\infty} \gamma_{n}^{\prime \prime}<\infty$, where $\alpha_{n}, \alpha_{n}^{\prime}$, and $\alpha_{n}^{\prime \prime}$ are three sequences in $[\varepsilon, 1-\varepsilon]$, for some $\varepsilon>0$. If $F=F\left(T_{1}\right) \cap F\left(T_{2}\right) \cap F\left(T_{3}\right) \neq \varnothing$, then $\left\{x_{n}\right\}$ converges strongly to a common fixed point of $T_{1}, T_{2}$, and $T_{3}$.

Proof. Since every nonself asymptotically nonexpansive mapping is uniformly $L$-Lipschitzian and nonself asymptotically quasi-non-expansive, the result can be deduced immediately from Theorem 3.3. This completes the proof. 
Theorem 3.5. Let $K$ be a nonempty closed convex subset of a real uniformly convex Banach space $E$. Let $T_{1}, T_{2}, T_{3}: K \rightarrow E$ be uniformly L-Lipschitzian and nonself asymptotically quasi-non-expansive mappings with sequences $\left\{r_{n}^{(i)}\right\}$ such that $\sum_{n=1}^{\infty} r_{n}^{(i)}<\infty$, for all $i=1,2,3$. Suppose that $\left\{x_{n}\right\}$ is defined by (1.6) with $\sum_{n=1}^{\infty} \gamma_{n}<\infty, \sum_{n=1}^{\infty} \gamma_{n}^{\prime}<\infty$, and $\sum_{n=1}^{\infty} \gamma_{n}^{\prime \prime}<\infty$, where $\alpha_{n}, \alpha_{n}^{\prime}$, and $\alpha_{n}^{\prime \prime}$ are three sequences in $[\varepsilon, 1-\varepsilon]$, for some $\varepsilon>0$. If $F=F\left(T_{1}\right) \cap F\left(T_{2}\right) \cap F\left(T_{3}\right) \neq \varnothing$ and one of $T_{1}, T_{2}$, and $T_{3}$ is demicompact, then $\left\{x_{n}\right\}$ converges strongly to a common fixed point of $T_{1}, T_{2}$, and $T_{3}$.

Proof. Without loss of generality, we may assume that $T_{1}$ is demicompact. Since $\lim _{n \rightarrow \infty} \| x_{n}-$ $T_{1} x_{n} \|=0$, there exists a subsequence $\left\{x_{n_{j}}\right\} \subset\left\{x_{n}\right\}$ such that $x_{n_{j}} \rightarrow x^{*} \in K$. Hence, from (3.39), we have

$$
\left\|x^{*}-T_{i} x^{*}\right\|=\lim _{n \rightarrow \infty}\left\|x_{n_{j}}-T_{i} x_{n_{j}}\right\|=0, \quad i=1,2,3 .
$$

This implies that $x^{*} \in F$. By the arbitrariness of $p \in F$, from Lemma 3.1, and taking $p=x^{*}$, similarly we can prove that

$$
\lim _{n \rightarrow \infty}\left\|x_{n}-x^{*}\right\|=d,
$$

where $d \geq 0$ is some nonnegative number. From $x_{n_{j}} \rightarrow x^{*}$, we know that $d=0$, that is, $x_{n} \rightarrow x^{*}$. This completes the proof.

Corollary 3.6. Let $K$ be a nonempty closed convex subset of a real uniformly convex Banach space $E$. Let $T_{1}, T_{2}, T_{3}: K \rightarrow E$ be nonself asymptotically nonexpansive mappings with sequences $\left\{r_{n}^{(i)}\right\}$ such that $\sum_{n=1}^{\infty} r_{n}^{(i)}<\infty$, for all $i=1,2,3$. Suppose that $\left\{x_{n}\right\}$ is defined by (1.6) with $\sum_{n=1}^{\infty} \gamma_{n}<\infty$, $\sum_{n=1}^{\infty} \gamma_{n}^{\prime}<\infty$, and $\sum_{n=1}^{\infty} \gamma_{n}^{\prime \prime}<\infty$, where $\alpha_{n}, \alpha_{n}^{\prime}$, and $\alpha_{n}^{\prime \prime}$ are three sequences in $[\varepsilon, 1-\varepsilon]$, for some $\varepsilon>0$. If $F=F\left(T_{1}\right) \cap F\left(T_{2}\right) \cap F\left(T_{3}\right) \neq \varnothing$ and one of $T_{1}, T_{2}$, and $T_{3}$ is demicompact, then $\left\{x_{n}\right\}$ converges strongly to a common fixed point of $T_{1}, T_{2}$, and $T_{3}$.

\section{Acknowledgments}

The authors would like to thank the referee and the editor for their careful reading of the manuscript and their many valuable comments and suggestions. This paper was supported by the National Natural Science Foundation of China (Grant no. 10671145).

\section{References}

[1] W. R. Mann, "Mean value methods in iteration," Proceedings of the American Mathematical Society, vol. 4, no. 3, pp. 506-510, 1953.

[2] S. Ishikawa, "Fixed points and iteration of a nonexpansive mapping in a Banach space," Proceedings of the American Mathematical Society, vol. 59, no. 1, pp. 65-71, 1967.

[3] K. K. Tan and H. K. Xu, "Approximating fixed points of nonexpansive mappings by the Ishikawa iteration process," Journal of Mathematical Analysis and Applications, vol. 178, no. 2, pp. 301-308, 1993.

[4] J. Schu, "Weak and strong convergence to fixed points of asymptotically nonexpansive mappings," Bulletin of the Australian Mathematical Society, vol. 43, no. 1, pp. 153-159, 1991.

[5] Q. H. Liu, "Iterative sequences for asymptotically quasi-nonexpansive mappings with error member," Journal of Mathematical Analysis and Applications, vol. 259, no. 1, pp. 18-24, 2001.

[6] N. Shahzad and A. Udomene, "Approximating common fixed points of two asymptotically quasinonexpansive mappings in Banach spaces," Fixed Point Theory and Applications, vol. 2006, Article ID 18909, 10 pages, 2006.

[7] C. E. Chidume, E. U. Ofoedu, and H. Zegeye, "Strong and weak convergence theorems for asymptotically nonexpansive mappings," Journal of Mathematical Analysis and Applications, vol. 280, no. 2, pp. 364-374, 2003. 
[8] L. Wang, "Strong and weak convergence theorems for common fixed point of nonself asymptotically nonexpansive mappings," Journal of Mathematical Analysis and Applications, vol. 323, no. 1, pp. 550-557, 2006.

[9] M. A. Noor, "New approximation schemes for general variational inequalities," Journal of Mathematical Analysis and Applications, vol. 251, no. 1, pp. 217-229, 2000.

[10] R. Glowinski and P. Le Tallec, Augmented Lagrangian and Operator Splitting Methods in Nonlinear Mechanics, vol. 9 of SIAM Studies in Applied Mathematics, SIAM, Philadelphia, Pa, USA, 1989.

[11] B. Xu and M. A. Noor, "Fixed-point iterations for asymptotically nonexpansive mappings in Banach spaces," Journal of Mathematical Analysis and Applications, vol. 267, no. 2, pp. 444-453, 2002.

[12] Y. J. Cho, H. Zhou, and G. Guo, "Weak and strong convergence theorems for three-step iterations with errors for asymptotically nonexpansive mappings," Computers E Mathematics with Applications, vol. 47, no. 4-5, pp. 707-717, 2004.

[13] S. Plubtieng, R. Wangkeeree, and R. Punpaeng, "On the convergence of modified Noor iterations with errors for asymptotically nonexpansive mappings," Journal of Mathematical Analysis and Applications, vol. 322, no. 2, pp. 1018-1029, 2006.

[14] H. F. Senter and W. G. Dotson Jr., "Approximating fixed points of nonexpansive mappings," Proceedings of the American Mathematical Society, vol. 44, no. 2, pp. 375-380, 1974.

[15] J. Schu, "Iterative construction of fixed points of strictly pseudocontractive mappings," Applicable Analysis, vol. 40, no. 2-3, pp. 67-72, 1991. 